\title{
LV NRW: „Gesundheitsberufe stärken - Fachkräftemangel entgegenwirken“
}

Mit diesem einhelligen Statement endete die interessante Diskussion mit den Politikern der Landtagsfraktionen während der Fachtagung des BLGS NRW am 27.10.2016 in den Westfalenhallen Dortmund mit 100 Teilnehmenden. Der Schwerpunkt lag auf den Wahlprüfsteinen für die Landtagswahl 2017. Hierzu wurde mit Oskar Burkert (CDU), Angela Lück (SPD), Arif Ünal (Grüne), Susanne Schneider (FDP) und Dennis Deutschkämmer (Piraten) zu unterschiedlichen Themen diskutiert.

Während sich nur die CDU innerhalb dieser Diskussion klar für eine Pflegekammer NRW aussprach, hielten sich die anderen Parteien bezüglich dieser Thematik (u.a. aufgrund der Pflichtmitgliedschaft) zurück. Oskar Burkert war es auch, der die Pflegenden zu einem "Aufschrei" aufforderte, um sich für die Verkammerung einzusetzen. Bezüglich des Pflegeberufegesetzes war es erneut die CDU, die sich klar dafür aussprach, während sich die SPD, die Grünen und auch die Piraten nicht deutlich positionieren konnten. Lediglich Susanne Schneider von der FDP sprach sich eindeutig gegen die generalistische Ausbildung aus.

Begonnen hat die interessante Fachtagung mit Grußworten des Landesvorstands (Thomas Kutschke) und des Bundesvorstands (Jochen Vennekate). Der eindrucksvolle Fachvortrag zum Thema: „Marketing an Pflegeschulen“ wurde von Dr. German Quernheim gehalten. Er berichtete vom „Emo-Faktor", der dazu führt, dass Lernende sich an einer Bildungseinrichtung wohl fühlen und folglich das Marketing durch deren Begeisterung unterstützen kann. Danach berichtete Dr. Christiane Riesner über Aktuelles aus dem Ministerium für Gesundheit, Emanzipation, Pflege und Alter (MGEPA). Ernüchternd war dabei der Umstand, dass 2.290 Ausbildungsplätze in der Pflege fehlen (Landesberichterstattung 2015). Aus diesem Grund wird erfreulicherweise das Umlageverfahren für die Ausbildungsplätze in der Altenpflege fortgeführt.

Innerhalb der Landesberichterstattung wird deutlich, dass die Zahl der Pflegebedürftigen in NRW von 485.000 im Jahr 2009 auf $581.000 \mathrm{im}$ Jahr 2013 gestiegen ist - eine Steigerung um 100.000 in vier Jahren. Das Land NRW hat in seinen Hochrechnungen kalkuliert, dass es im Jahr 2030 rund 700.000 Pflegebedürftige geben wird. Diese Prognose erscheint bei den aktuellen Zahlen absolut unrealistisch. Die Anzahl dürfte weit höher liegen. Erschreckend ist auch, in welchem Maß der Anteil der Vollzeitbeschäftigten in

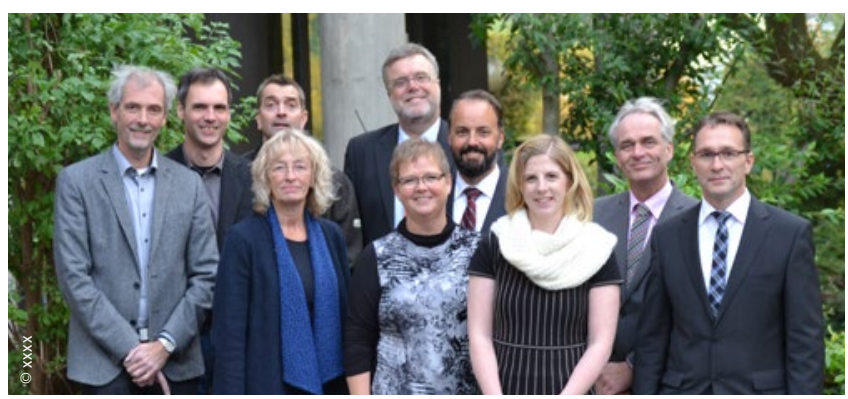

Anlässlich der Landesversammlung trafen sich Regionalgruppensprecher/innen und Vorstand (vInr, hinten): Torsten Edelkraut, Gregor Pleiss, Dirk Bross, Thomas Kutschke, Stefan Völkert (vInr, vorn): Andreas Winter, Monika Cremer-Biermann, Monika Huth, Daniela Schlosser, Klaus Stinner der Pflege abgenommen hat: 2013 lag er in ambulanten Einrichtungen nur noch bei 34,5\% und im stationären Bereich bei 38,2\%. Dadurch wird der Fachkräftemangel erheblich verschärft.

Bei der Landesversammlung NRW wurde Monika Huth als stellvertretende Vorsitzende bestätigt, da Andreas Riekötter aus persönlichen Gründen zurückgetreten war. Ebenfalls bestätigt wurde Stephan Völkert, der neu in den Landesvorstand berufen worden war.

\section{Eine Pflegekammer für NRW}

Weitere Tätigkeiten des BLGS NRW sind auf die Etablierung einer Pflegekammer gerichtet. In Zusammenarbeit mit dem Landespflegerat startete eine Initiative, die zum Ziel hat, die Pflegenden über die Pflegekammer zu informieren. Derzeit werden Multiplikatoren geschult, die für Informationen über die Pflegekammer NRW zur Verfügung stehen.

In diesem Zusammenhang fand ein Expertengespräch am 13. September $2016 \mathrm{im}$ Landtag statt, zu dem Thomas Kutschke als Experte geladen war. Bei der Anhörung im Landtag am 26.10.2016 war auch der Pflegerat NRW mit Ludger Risse, Jens Albrecht und Thomas Kutschke vertreten. Die bekannten Positionen von Befürwortern und Gegnern wurden gehört. Diese Anhörung stellte klar, dass das Auftreten der verschiedenen Experten verdeutlicht, warum die Pflege eine Kammer - und damit eine einheitliche professionelle Stimme - braucht. Insgesamt wurden die Argumente zur Gründung einer Pflegekammer sachlich und faktenorientiert vorgetragen. Zu einer endgültigen Entscheidung wird es sicherlich erst nach den Landtagswahlen kommen. Bereits jetzt zeichnet sich ab, dass es eine Befragung der Pflegenden geben soll. Daher ist die Schulung von Multiplikatoren aus den Reihen des BLGS absolut notwendig.

\section{Altenpflegeausbildung}

Wie bereits berichtet, wurde für die Qualitätssicherung der Ausbildung in der Altenpflege eine Arbeitsgruppe vom MGEPA einberufen, um Qualitätsstandards zu definieren. Der BLGS ist aufgrund der geplanten Festlegung einer Lehrer-Schüler-Relation von 1:50 bereits aus dieser Arbeitsgruppe ausgetreten. Aktuell hat das MGEPA die Arbeit eingestellt und dies mit der Generalistikdiskussion und der damit verbundenen unklaren Zukunft der Altenpflegeausbildung begründet.

\section{Auftakt Praxisanleiterkonferenz}

Am 27. September 2016 fand die erste Praxisanleiterkonferenz im Regierungsbezirk Düsseldorf statt. Mit 22 Teilnehmern war das Interesse sehr hoch. Andrea Jaworski, Augusta Krankenhaus Düsseldorf, und Ralf Steffen, Lukas Krankenhaus Neuss, werden zukünftig die Praxisanleiterkonferenz koordinieren.

\section{Landespflegerat NRW}

Im September wurde der Vorstand des Landespflegerats neu gewählt. Aus dem BLGS wurde Thomas Kutschke als Stellvertreter wiedergewählt.

Daniela Schlosser

BLGS-Landesvorstand Nordrhein-Westfalen 